\title{
Passive Clustering for Efficient Energy Conservation in Wireless Sensor Network
}

\author{
Abderrahim MAIZATE \\ STIC Laboratory \\ Chouaib Doukkali University, B.P.:20 \\ Eljadida, Morocco
}

\author{
Najib EL KAMOUN \\ STIC Laboratory \\ Chouaib Doukkali University, B.P.:20 \\ Eljadida, Morocco
}

\begin{abstract}
A wireless sensor network is a set of miniature nodes that consume little energy and route information to a base station. It will enable reliable monitoring of a wide variety of phenomena for civilian, military and medical applications. Almost any sensor network application requires some form of self-organisation to route information. Recent years many protocols for network self-organization and management have been proposed and being implemented. Hierarchical clustering algorithms are very important in increasing the network's life time. The most important point in this algorithm is cluster head selection and cluster formation because a good clustering guarantees reliability, energy efficiency and load balancing in the network. In this paper, we will use the principles of passive clustering to propose a new mechanism for selecting clusterheads. This mechanism allows the election of an alternate for each cluster head and a dynamic balancing of the role of clusterhead to the alternate when leaving or failure. Thus, it provides several advantages network reliability, stability of clusters and reduces energy consumption among the sensor nodes. Comparison with the existing schemes such as Passive Clustering and GRIDS (Geographically Repulsive Insomnious Distributed Sensors) reveals that the mechanism for selecting an alternate for clusterhead nodes, which is the most important factor influencing the clustering performance, can significantly improves the network lifetime.
\end{abstract}

Keywords-wireless sensor networks; self-organization; Clustering passive; Clustering; network lifetime; energy efficiency; Fault tolerance; Residual Energy.

\section{INTRODUCTION}

The wireless sensor networks (WSN) is currently generating a growing interest among researchers. A WSN can be generally described as a network of nodes that cooperatively sense and may control the environment enabling interaction between persons or computers and the surrounding environment [1]. Today, due to recent advances in wireless technologies, new products operating wireless sensor networks are used to retrieve data from these applications. Examples include environmental monitoring, smart homes and offices, surveillance, intelligent transportation systems, and many others (Fig 1).

The use of wireless sensor networks (WSNs) does not require a specific infrastructure. But it poses a problem of scalability, energy conservation and connectivity over time [1], [2], [3]. Also wireless sensor networks can be exposed to

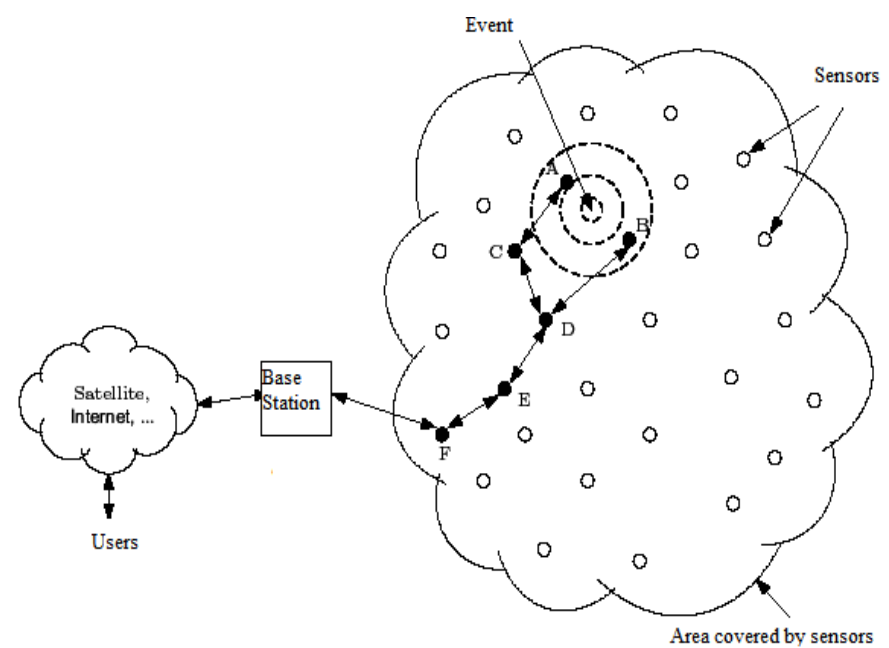

Fig. 1. Traditional scheme of a wireless sensor network.

highly dynamic and mobile environments, and therefore they must be dynamic and mobile environments, and therefore they must be fault tolerant nodes. Algorithms for wireless sensor networks must be distributed to avoid single point's failure, and self-organization for scalable deployment.

Self-organization can be defined as the emergence of a global behavior from local interaction [4]. Wireless sensor networks are bandwidth and energy constrained. Selforganization algorithms that minimize the number of message transmissions (and receptions) are preferable. The challenge is to organize dynamic and spontaneous nodes to form a network and prolonging the life-time, while satisfying the constraints of service quality. It is therefore necessary for self-organizing algorithms proposed to limit control packet exchanges for a minimum expenditure of energy and preserve the structure of self-organization for better stability and reliability the network.

How to design an energy efficient protocol to lessen the battery consumption and prolong the network lifetime becomes a critical issue. The passive clustering structure is a way to reduce the energy consumption and can used to perform data aggregation, the process involves grouping nodes into clusters and elect one cluster head $(\mathrm{CH})$ for each cluster to transmit the collected data to central base station through other $\mathrm{CHs}$. Obviously, we can save a great amount of unnecessary transmission by such clustering and data aggregation mechanisms. 
A number of recent clustering approach provide techniques to balance energy consumption of network nodes, these techniques are essentially turning the role of $\mathrm{CH}$ randomly but it is not fault tolerant nodes especially nodes clusterheads.

The algorithm presented in this paper considers nodes Clusterhead. In this algorithm, we rely on passive clustering to create a redundant role for clusterhead. The node that replaces the cluster head will be the state clusterhead alternate. The clusterhead and the alternate shall periodically to transmit information and verify the presence of each. The proposed algorithm is designed for generating a reliable, scalable and efficient topology, reducing consumption of scarce resources during query dissemination and, thereby extend network lifetime.

The rest of the paper is organized as follows. In Section II, the term self-organization in wireless sensor network is discussed as well as related concepts. We present a description and analysis of the proposed algorithm in Section III, followed by simulation results in Section IV. The last section concludes the paper.

\section{RELATED WORK}

Clustering is an important research topic in the areas of wireless sensor network (WSN) because clustering improves the performance of many systems. In WSN, clustering can be used to improve the network performance through quality of service metrics such as throughput and delay, in the presence of both mobility and a large number of mobile nodes with minimal resources. We have investigated a number of prior works which consider the wireless sensor network selforganization with clustering mechanisms, a large variety of approaches have been presented by various researchers $[5,6$, $7,8,9,10,11,12,13,14,14]$.

Passive clustering [15] can be described as on demand cluster formation protocol that does not use dedicated protocolspecific control packets or signals. The formation of cluster is dynamic and initiated by the first data message to be flooded. Which in turn reduces the duration of the initial set-up period, and the benefits of the reduction of the forwarding set can be felt by calculating the total energy consumed because the main function of the clusters is to optimize the exchange of flooded messages.

In passive clustering each node operates the MAC sender address carried by the received packets to collect neighbor information, and can construct clusters even without collecting the complete neighbor list. Instead of using protocol specific signals or packets, passive clustering reserves two bits for the following four states of a mobile node: :

( 1) Initial, (2) Cluster head, (3) Gateway and (4) Ordinary. At the beginning, every sensor node is in the INITIAL state until it receives a packet. If the sender's packet is not CLUSTERHEAD, this sensor node switches to CLUSTERHEAD-READY. This node will become a CLUSTERHEAD if it successfully transmits a packet before receiving any packets from others. If the sensor node receives a packet from a CLUSTERHEAD it changes state to ORDINADRY. Any sensor node that hears more then one CLUSTERHEAD becomes GATEWAY.
Passive clustering has several mechanisms for the cluster formation such as: Gateway Selection Heuristic and First Declaration Wins rule. The Gateway Selection Heuristic provides a procedure to elect the minimal number of gateways required to maintain the connectivity between clusterheads. With the First Declaration Wins rule, a node that first transmits a data message will be a clusterhead of the rest of nodes in its clustered area.

Passive clustering maintains clusters using implicit timeout. A node assumes that some nodes are out of clustered area if they have not sent any data longer than timeout duration. With reasonable offered load, a node can catch dynamic topology changes.

In [16], an interesting technique has been proposed "Energy Conserving Passive Clustering (ECPC) algorithm" which takes account of both residual energy and distance for becoming cluster head and gateway and also eliminate the problem of idle listening through periodic sleep and awake among the cluster members. This algorithm outperforms Directed Diffusion (DD) [17] and Passive Clustering Directed Diffusion (PCDD) [18] in terms of energy dissipation and network lifetime. It also generates much less gateway nodes than PCDD algorithm. The selection of the clusterhead is based on the higher residual energy with in the 1-hop neighbours and the distance to form better clusters. The gateway selection procedure prioritizes the residual energy of the node which wants declare itself as a gateway and average distance of clusterhead nodes with in -hop.

Some of the well known clustering protocols are the LowEnergy Adaptive Clustering Hierarchy (LEACH) [19] and the Hybrid-Energy-Efficient Distributed (HEED) [20]. Both of which are self-organizing, and distributed protocols. LEACH achieves energy saving in three ways: randomized rotation of cluster head, sleep mode and data aggregation. CHs are randomly selected. The decision of CHs is simply based on the suggested percentage of them for the network and the number of times the node has been a $\mathrm{CH}$ therefore two ch can be selected in close area, thus the system efficiency may be decreased. There are several variant of this algorithm such as: LEACH-B, LEACH-C, LEACH-E and M-LEACH.

HEED improves network lifetime over LEACH by distributing energy consumption. HEED focuses on choose appropriate $\mathrm{CHs}$ by using residual energy as the primary clustering parameter to select a number of tentative CHs. Those tentative CHs inform their neighbours of their intentions to become CHs. These advertisement messages include a secondary cost measure that is a function of neighbor proximity or node degree. This secondary cost is used to help the regular nodes in choosing the best clusterhead to join, and to avoid elected $\mathrm{CHs}$ being within the same cluster area of each other. If a $\mathrm{CH}$ is far from the sink, it tries to send the aggregate data to another $\mathrm{CH}$ instead of sending to the sink directly.

GRIDS [21] is an energy-aware cluster formation protocol which increase network lifetime by using an efficient selection mechanism of critical (or not) nodes. This mechanism allows balanced energy consumption among the sensor nodes without requiring additional overheads including additional signaling, time synchronization and global information. GRIDS is based 
on an energy model which delivers node's remaining energy level in real time. This information is piggybacked in the nodes packet header. Each sensor determines being insomnious or not based on its remaining energy and the number of neighbouring insomnious nodes and their energy level. An efficient flooding during each wake up period determines insomnious nodes in the network.

GRIDS inherit PC for constructing and maintaining clusters. The most important advantage of GRIDS compared to $\mathrm{PC}$ is that a set of nodes in a cluster with higher energy levels have higher probability to become critical nodes, i.e., $\mathrm{CH}$ or GW. In Passive Clustering, CHs keep their cluster status until there is a $\mathrm{CH}$ collision, i.e. the hop distance between two $\mathrm{CHs}$ becomes 1 , and one of them resigns from $\mathrm{CH}$. In GRIDS, an energy abundant node can challenge $\mathrm{CH}$ and usurps the role. Even if there is a $\mathrm{CH}$ declaration, nodes can challenge when their energy levels are higher than the one of $\mathrm{CH}$. These nodes keep their cluster status even if they receive packets from the current $\mathrm{CH}$.

Clustering stability, fast convergence time, the consumption of energy and mobility of nodes are important properties required of clustering algorithms. To improve the stability and reliability of clustering and reduce consumption of energy, we developed a new rule of electing alternates of clusterheads that represent critical nodes for passive clustering.

\section{PROPOSAL- PASSIVE CLUSTERING FOR EFFICIENT}

\section{ENERGY CONSERVATION IN WIRELESS SENSOR NETWORK}

In this section, we present the details of new algorithm. The advantage is that improves reliability of the network by selecting an alternate for clusterhead, uses balanced energy consumption among network nodes and keeps longer the structure of clusters.

As a result, the network stability and reliability are preserved, the transmission delay is decreased and the life time of the network is significantly increased.

\section{A. PCEEC mechanism}

PCEEC (Passive Clustering for Efficient Energy Conservation in Wireless Sensor Network) defines a protocol for cluster formation and election of alternates of the clusterheads based on the following principles:

1) There are six possible states: dead, initial, ordinary, clusterhead_ready, custerhead, gateway and clusterheadalternate

2) Initially or when there are no networks activities for a long time, all nodes are in the 'initial' state. This state does not change as long as a node does not receive a packet from another node.

3) When a node receives a packet and if the state of a sender is not ClusterHead, the receiver's state.

4) switches to ClusterHead_ready, otherwise the node switches to state ordinary or gateway.
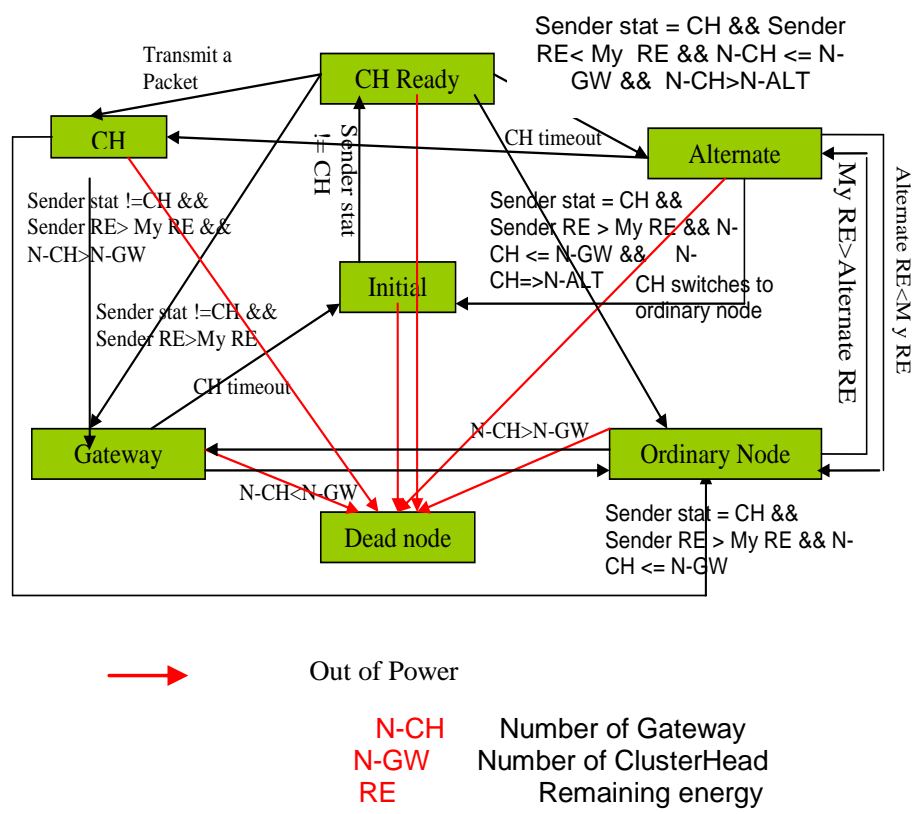

Fig. 2. State diagramme of PCEEC

5) The node ClusterHead_ready switches to state gateway when the number of ClusterHeads is greater or equal to the number of Gateways. Otherwise, the node becomes an Ordinary Node or an alternate node.

6) The node ClusterHead_ready switches to state alternate when the number of ClusterHeads is greater or equal to the number of Gateways and the number of ClusterHeads is greater to the number of alternates. Otherwise, the node becomes an Ordinary Node.

7) The clusterHead chooses the node with the highest level of energy as an alternate on failure the previous. The clusterHead checks periodically the presence of his alternate. In the case of the leaving or failure of the alternate, the cluster head rerun the selection process of a new alternate.

8) Similarly, if the alternate discovers the leaving or failure of cluterhead it switches to state ClusterHead and launch the procedure to select an alternate (see Figure 2).

9) An ordinary node switches to alternate if its energy is higher. The alternate node switches to state ordinary.

PCEEC uses the same principles as PC for the construction and maintenance of clusters in wireless sensor networks. It also inherits the characteristics of the algorithm GRIDS by giving nodes with the highest level of energy to become a critical node, i.e., ClusterHead, Alternate or GateWay.

\section{B. Operational Description}

In each cluster, we will have a cluster head that centralizes information and an alternate to replace him on failure. Thus the structure of the cluster will be further preserved. 
TABLE I.

A PSEUDO-CODE THAT SHOWS THE OPERATION DETAILS OF PCEEC.

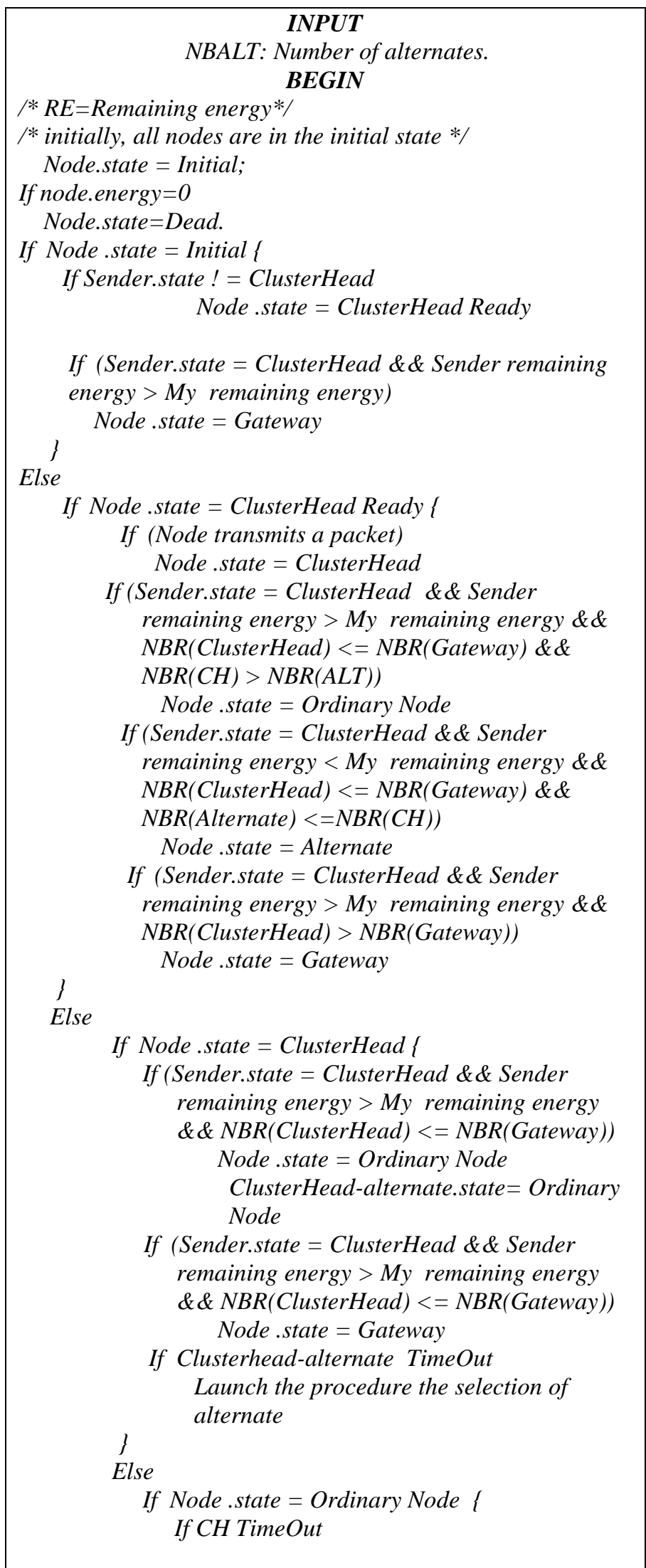

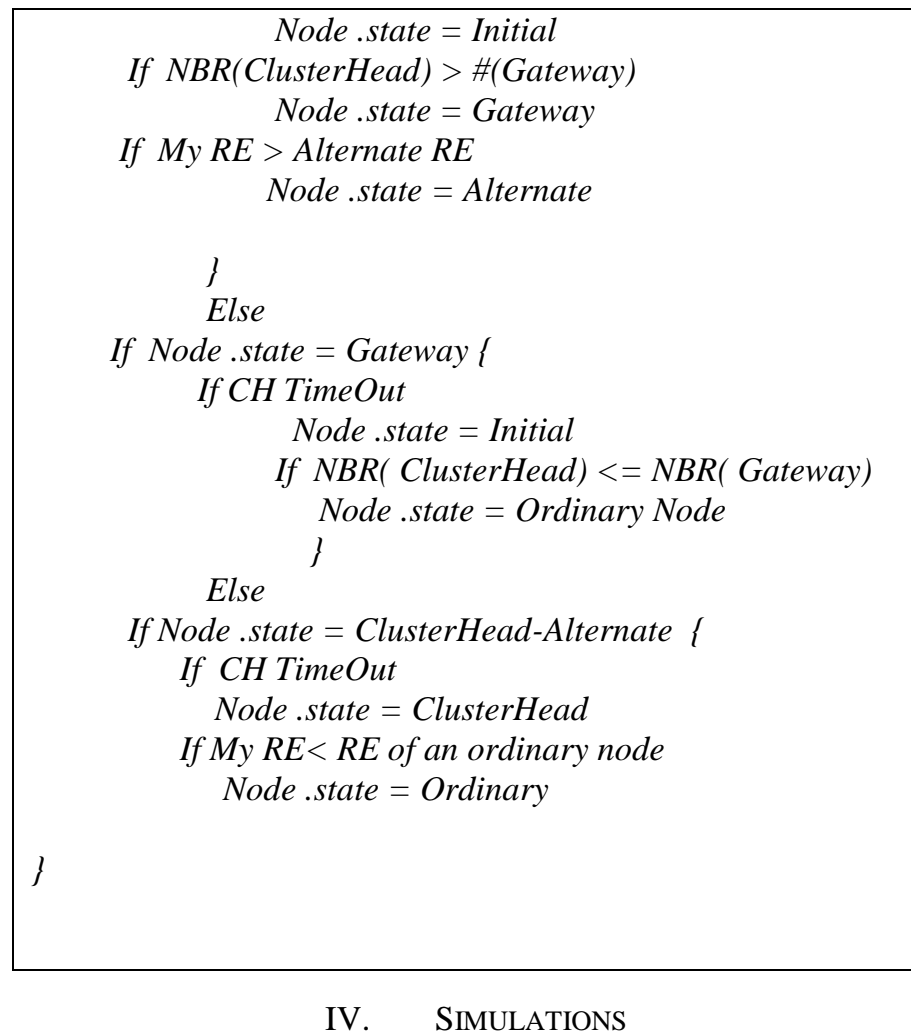

In this section, we present comparison between proposed algorithms and two most important clustering protocols, PC and GRIDS. This comparison is evaluated using the simulator GLOMOSIM [22], which is a scalable simulation environment for wireless networks based on the Parsec language [23]. We begin first by specifying the metrics that we considered interesting to evaluate this algorithm and results obtained.

A. Metric

To evaluate the performance of PCEEC protocol, we will use the following parameters:

- Lifetime of the network: duration until the death of the last node in the network.

- Delivery rate: it characterizes the routing performance and is based on network conditions. It is the fraction effective throughput / maximum flow.

- Energy consumption: Amount of energy needed to sustain the network during its lifetime and data collection.

- Dead Nodes: Represents the percentage of dead nodes over time in the wireless sensor network.

B. Simulation scenarios

The simulation parameters used are as follows:

- the roaming space is $500 \times 500 \mathrm{~m}$ square,

- The radio propagation of each node reaches up to 250 meters

- The channel capacity is 2 Mbits/second.

- The battery capacity is equal to $500 \mathrm{~mW}$ 
- Simulations use a variable number of nodes; distributed randomly in the roaming area;

- The random-way point model is used for node mobility

- The traffic model used is constant bit rate (CBR). Packet length is 566 bytes.

- Each node sends 100 packets with inter-arrival time of 0.2 second.

- Number of nodes: 300.

- AODV [24] is chosen as the routing protocol;

We use four metrics for analyze and compare the simulation results: network lifetime, consumed energy, Percentage of dead nodes and delivery ratio at base station.

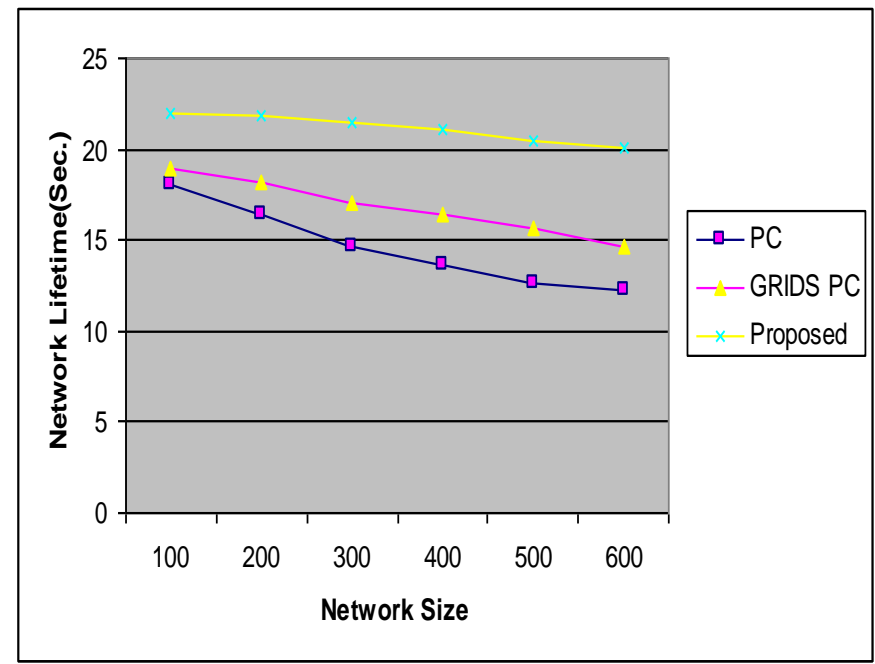

Fig. 3. Network lifetime of PCEEC compared to PC and GRIDS PC.

Figure 3 plots the network size and the network lifetime for the three protocols. We measure the network lifetime when the number of sensors varies between 50 and 600 . The energy consumption model is linear. The results show that PCEEC achieves better performances compared to the two others. The PCEEC protocol improves the network lifetime performance, and the gain in lifetime increases with the size of the network. Thus, we conclude that this protocol is more suitable for large scale networks.

Figure 4 shows that the proposed algorithm consumes less energy than the passive clustering and GRIDS PC as can be seen. The PCEEC protocol saves $20 \%$ of the total energy consumed, because our algorithm preserves better cluster structure by preventing reinitialization in case of the leaving or failure of a clusterhead.

Figure 5 indicates the energy consumption, during the phase of reclustering, generated by PC, GRIDS PC and proposed PCEEC algorithm in case the failure the node clusterhead. It is obviously that the proposed algorithm reduces significantly the consumed energy with a percentage very close to $50 \%$. This gain is due to the preservation of the cluster structure.

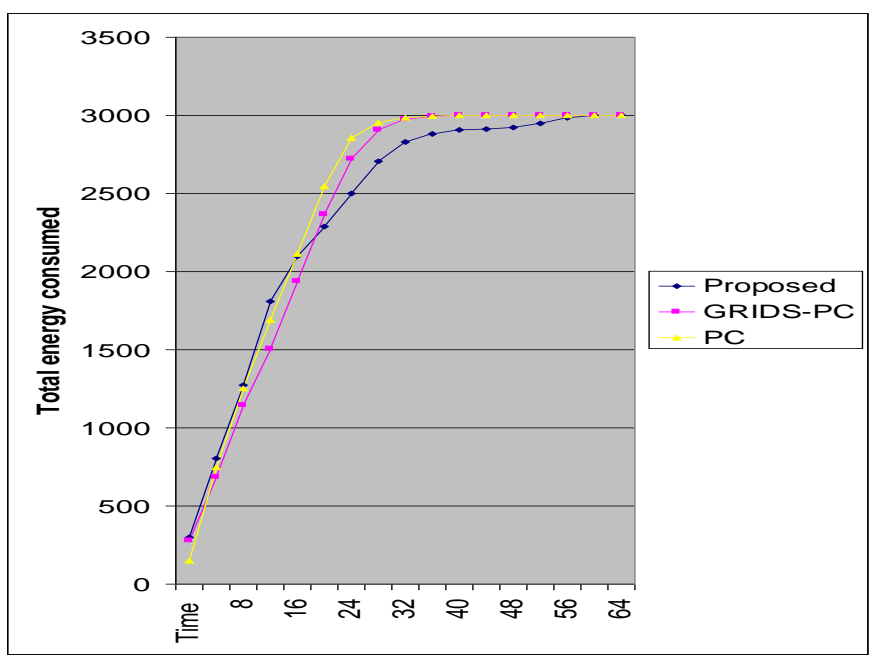

Fig. 4. Total energy consumed by PCEEC compared to PC and GRIDS PC.

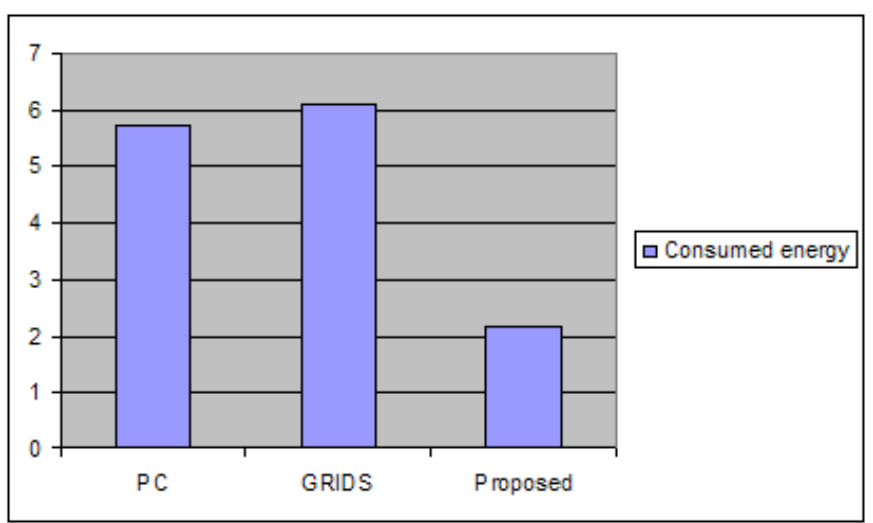

Fig. 5. Consumed energy by the reclustering after the failure of the $\mathrm{CH}$ in a simulation of 300 nodes.

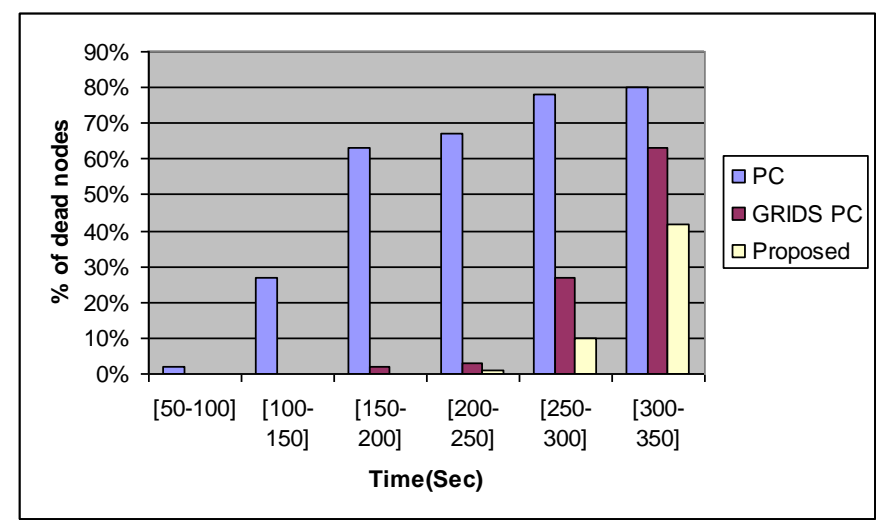

Fig. 6. Percentage of dead nodes in a simulation of 300 nodes.

Figure 6 shows dead nodes ratio as a function of time. PCEEC outperforms and achieves better results in optimizing the energy consumption compared to passive clustering and GRIDS PC.

Similarly, Fig. 7 shows that also the Delivery ratio is much better with PCEEC, because PCEEC decreases the number of dead nodes and retains more the cluster structure. 


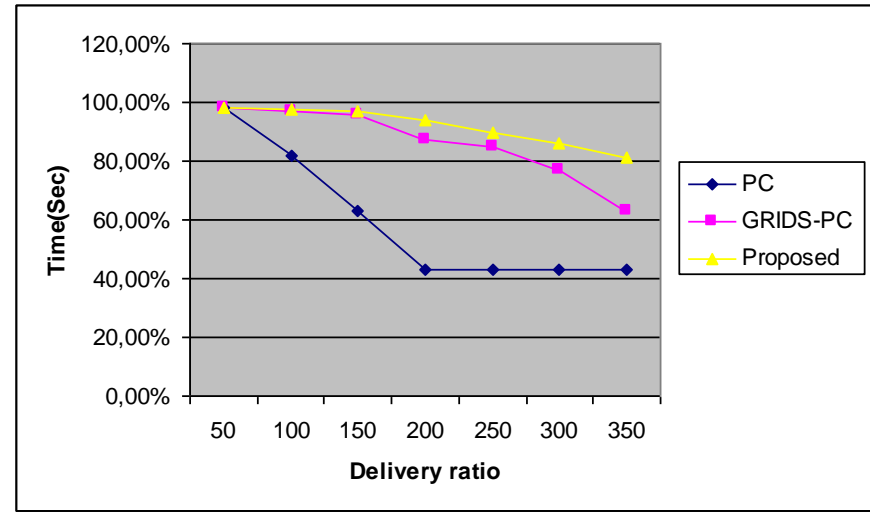

Fig. 7. Delivery ratio in a simulation of 300 nodes.

Thus, the simulation results show that the Passive Clustering for Efficient Energy Conservation in Wireless Sensor Network scheme not only provides an efficient forwarding and balances the energy consumption but also improves network performance.

\section{CONCLUSION AND FUTURE WORK}

We introduce a passive clustering mechanism for electing clusterheads and clusterheads alternate in wireless sensor networks. The selection of cluster heads and alternate is performed according to the remaining energy of sensor nodes. The sensor nodes with the highest energy in the clusters can be a cluste headsand alternates at different cycles of time. Thus, the role of cluster heads and alternate can be switched dynamically. Simulation results show the effectiveness of the approach in reducing the amount of energy consumed by the network in comparison with two well-known protocols, passive clustering and GRIDS PC.

In the future, it is planned to provide enhancements to the proposed algorithm to make decisions using distance during the selection of clusterheads and alternates to improve the performance further.

\section{REFERENCES}

[1] Verdone, R.; Dardari, D.; Mazzini, G.; Conti, A. Wireless Sensor and Actuator Networks; Elsevier: London, UK, 2008.

[2] Akyildiz I. F., Y. Sankarasubramaniam W. Su. et Cayirici E. Wireless sensor networks : a survey. Computer Networks (Elsevier), vol. 38(4), pp. 393-422, March. 2002.

[3] Yick J., Mukherjee B. et Ghosal D. Wireless sensor network survey. Computer Networks (Elsevier), vol. 52, $\mathrm{n}^{\circ} 12$, pp. 2292-2330, Aug. 2008.

[4] W. Heinzelman, A. Chandrakasan, and H. Balakrishnan, "Energyefficient communication protocol for wireless microsensor networks," in Proc. of the 33rd Annual Hawaii International Conference on System Sciences (HICSS'00), Hawaii, USA, Jan. 2000, pp. 30053014.

[5] Houda Zeghilet; Moufida Maimour; Nadjib Badache; Francis Lepage. On the Use of Passive Clustering in Wireless Video Sensor Networks Int. J. of Sensor Networks, 2012 Vol.11, No.2, pp.67-80.

[6] Ketki Ram Bhakare,R. K. Krishna,Samiksha Bhakare, "An Energyefficient Grid based Clustering Topology for a Wireless Sensor Network," International Journal of Computer Applications. Volume 39No.14, February 2012.
[7] Rajnish Kansal, "Enhanced Uniform Distributed Clustering Algorithm (UDCA) In Wireless Sensor Network," International Journal of Emerging Technology and Advanced Engineering, Volume 2, Issue 6, June 2012.

[8] Zhu Yonga, Qing Peia, "A Energy-Efficient Clustering Routing Algorithm Based on Distance and Residual Energy for Wireless Sensor Networks," 2012 International Workshop on Information and Electronics Engineering (IWIEE).

[9] M. H. Tolou and J. Chitizadeh, "Lifetime prolonging of wireless sensor networks via a recursive clustering algorithm," in Proc. of the third IEEE International Conference in Central Asia on internet the Next generation of mobile,wireless and optical communications networks (IEEE/IFIP ICI'07 ), Tashkent, Sep. 2007, pp. 1-6.

[10] C. H. and M. S., "Cluster sizing and head selection for efficient data aggregation and routing in sensor networks," in Proc. of IEEE Wireless Communications and Networking Conference (IEEE WCNC'06), vol. 4, Las Vegas, NV, USA, 2006, pp. 2318-2323.

[11] Y. Zhou, M. Hart, S. Vadgama, and A. Rouz, "A hierarchical clustering method in wireless ad hoc sensor networks," in Proc. of the IEEE International Conference on Communications (IEEE ICC'07), Glasgow, Scotland, Jun. 2007, pp. 3503-3509.

[12] J. Yu, W. Liu, J. Song, and B. Cao, "Eemr: An energy-efficient multihop routing protocol for wireless sensor networks," in Proc. Of the International Conference on Computer Systems and Application (IEEE/ACS AICCSA'08), Doha, Qatar, Mar. 2008, pp. 291-298.

[13] M. H. Yeo, M. S. Lee, S. J. Lee, and J. S. Yoo, "Data correlation-based clustering in sensor networks," in Proc. of the International Symposium on Computer Science and its Applications (CSA'08), Hobart, Australia, Oct. 2008, pp. 332-337.

[14] Amir Akhavan Kharazian, K. Jamshidi and M. Khayyambashi, "adaptive clustering in wireless sensor network: considering nodes with lowest energy," International Journal of Ad hoc, Sensor \& Ubiquitous Computing (IJASUC) Vol.3, No.2, April 2012.

[15] M. Gerla, T.J. Kwon and G. Pei "On Demand Routing in Large Ad Hoc Wireless Networks with Passive Clustering", Proceedings of IEEE WCNC 2000, Chicago, IL, Sep. 2000.

[16] Md. Mamun-or-Rashid, M. Mahbub Alam and C. Seon Hong, "Energy Conserving Passive Clustering for Efficient Routing in Wireless Sensor Network,"

[17] C. Intanagonwiwat, R. Govindan, and D. Estrin, Directed diffusion: a scalable and robust communication paradigm for sensor networks, Proceedings of ACM MobiCom '00, Boston, MA, (2000) 56-67

[18] Handziski V., Köpke A., Karl H., Frank C. and Drytkiewicz W. "Improving the Energy Efficiency of Directed Diffusion Using Passive Clustering", in Proc of the 1st European Workshop on Wireless Sensor Networks (EWSN), LNCS 2920, Berlin, Germany, (2004) 172-187

[19] W. Heinzelman, A. Chandrakasan and H. Balakrishnan, EnergyEfficient Communication Protocol for Wireless Micro-sensor Networks, Proceedings of the 33rd Hawaii International Conference on System Sciences (HICSS '00), (2000)

[20] O. Younis and S. Fahmy, "HEED: A Hybrid, Energy-efficient, Distributed Clustering Approach for Ad Hoc Sensor Networks," IEEE Transactions on Mobile Computing, vol. 3, no. 4, pp. 366-379, 2004.

[21] El Ghanami, D. Kwon, T.J.; Hafid A., "GRIDS: Geographically Repulsive Insomnious Distributed Sensors - An Efficient Node Selection Mechanism Using Passive Clustering," Networking and Communications, 2008. WIMOB '08. IEEE International Conference on Wireless and Mobile Computing,

[22] M. Gerla, L. Bajai, M. Takai, and R. Ahuja. GloMoSim: A Scalable Network Simulation Environment, Technical Report 990027, University of California at Berkley, 1999.

[23] R. Bagrodia, R. Meyer, M. Takai, Y. Chen, X. Zeng, J. Martin, B. Park, H. Song, Parsec: A parallel simulation environment for complex systems, Computer, Vol. 31(10), October 1998, pp. 77-85.

[24] C. E. Perkins et al., Ad hoc on-demand distance vector (AODV) routing, [Online] Available: http://www.ietf.org/internet-drafts/draft-ietf-manetaodv-13.TX 